\title{
Transplantable programmed death ligand 1 expressing gastroids from gastric cancer prone $\mathrm{Nfkb} 1^{-/-}$mice
}

\author{
Jun T. Low ${ }^{1,2}$, Gwo-Yaw Ho (iD ${ }^{1,2,3}$, Mark Scott ${ }^{1,2,6}$, Chin Wee Tan (iD ${ }^{1,2}$, Lachlan Whitehead (iD ${ }^{1,2}$, Kathy Barber ${ }^{1,2}$, Hon Y. K. Yip (iD ${ }^{1,2,7}$, \\ Johanna F. Dekkers ${ }^{1,4,8}$, Yumiko Hirokawa ${ }^{1,2}$, John Silke $\mathbb{D}^{1,2}$, Antony W. Burgess ${ }^{1,2,5}$, Andreas Strasser $\mathbb{D}^{1,2}$, Tracy L. Putoczki ${ }^{1,2,5,9}$ and \\ Lorraine A. O'Reilly (iD ${ }^{1,2,9 凶}$
}

(c) The Author(s) 2021

Cell Death and Disease (2021)12:1091; https://doi.org/10.1038/s41419-021-04376-2

Gastric cancer (GC) is the fifth most common cancer and the third highest cause of cancer-related deaths globally [1]. There are several histological subtypes of GC [2], with intestinal type GC (IGC) the most common. IGC is initiated by inflammatory gastritis, often driven by Helicobacter pylori (H. pylori) or EBV infection [1]. This results in sustained activation of NF-KB transcription factors, which drive the expression of inflammatory factors thought to promote tumorigenesis [2]. Programmed death ligand 1 (PD-L1) is the ligand of the immune checkpoint regulator PD-1 that is expressed on T cells. The PD-L1/PD1 interaction inhibits cytotoxic T cell mediated killing of cancer cells [3]. Tumours can hijack this pathway, for example by expressing PD-L1, to render them resistant to such immune attack [3]. Immune checkpoint inhibitor therapy (ICIT) enhances the killing of malignant cells, including GC [4], by blocking PD1 or CTLA4 or the PD1 ligands, PDL1/PDL2.

We developed a mouse model of IGC that is driven by loss of NF-kB1, a member of the REL/NF-kB family of transcription factors. $N f k b 1^{-1-}$ mice present with abnormally increased expression of TNF and activation of STAT1 in the stomach, resulting in an inflammatory immune response that culminates in the development of GC $[5,6]$. Cells within the stomachs from pre-neoplastic $\mathrm{Nfkb}^{-1-}$ mice display abnormally increased proliferation, JAK/ STAT1 signalling and PD-L1 expression [5, 6] (Supplementary Fig. 1A). Pertinently, polymorphisms in human NFKB1 that diminish its function have been linked with increased risk for GC [6]. The EBV ${ }^{+}$ and Microsatellite Instability ${ }^{\text {hi }}$ (MSI ${ }^{\text {hi }}$ ) subtypes of human GC [7] exhibit features similar to the GC that arise in $N f k^{-1-}$ mice, suggesting that they are also driven by sustained inflammation, immune activation and that they may benefit from ICIT [6]. Organoids derived from human GC tissue and from animal models are an important experimental tool in GC research and for the testing of novel targeted therapies [8].

We generated gastric organoids (GOs) from the stomachs of both young, healthy and older tumour bearing $N \mathrm{fkb}^{-1-}$ mice (Fig. 1A, Supplementary 1B, Supplementary Tables 1, 2). Gastric organoids (GOs) derived from cells from the stomachs of young (7-8 week) wt or $N f k b 1^{-1-}$ exhibited spheroid-like, cyst-like and budding morphologies (Fig. 1A, B, Supplementary 2) as previously described [9]. Quantitative bright field microscopy revealed that there were no significant differences between the total numbers of organoids, budding (total numbers) or budding potential (frequencies) between $\mathrm{Nfkb}^{-/-}$and control wt GOs on days 4,6 or 8 of culture (Fig. 1C-F).

We also derived organoids from gastric tumours (TGOs) of

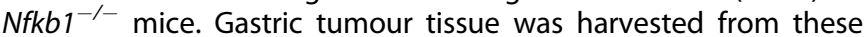
mice (>17 months, \#1391, 1392, 1399) for histological examination, flow cytometric analysis and organoid culture. Histological examination of these mice confirmed dysplasia and gastric invasion of neoplastic cells (Fig. 1G). Consistent with our findings in pre-neoplastic $N \mathrm{fkb}^{-1-}$ mice $[5,6]$, which showed abnormally elevated PD-L1 protein expression on myeloid and epithelial cells in the stomach compared to wt control mice, flow cytometric analysis revealed high levels of PD-L1 on both $\mathrm{CD} 11 \mathrm{~b}^{+}$myeloid cells and Epcam ${ }^{+}$epithelial cells in the stomachs of all GCburdened $\mathrm{Nfkb}^{-1-}$ mice (Supplementary Fig. 3A). TGOs were generated from each tumour sample and expanded as described above (Fig. 1H, Supplementary Tables 1, 2, Supplementary methods). All TGOs exhibited either spheroid, cystic or budding morphology (Fig. $1 \mathrm{H}$ ) and could be expanded for at least 30 passages ( 60-100 days, Supplementary Fig. 3B). Following establishment of the method for the passaging of TGOs, a biobank of TGO lines from $\mathrm{Nfkb}^{-1-}$ mice was initiated.

In all three independently derived $N f k b 1^{-1-}$ TGOs (\#1391, \#1392 and \#1399), PD-L1 expression remained high, even after repeated passaging and expansion in vitro (Fig. 1I). The TGOs were used for transplantation studies and injected subcutaneously into Nod-scid; common gamma chain ${ }^{\text {null }}$ (NSG) mice (Fig. 1J, Supplementary 3C). Within two months, tumour growth from TGO\#1399 was observed in recipient NSG mice and from TGO\#1391 and TGO\#1392 at later time points (Fig. 1J, K). Macroscopic analysis revealed cystic tumour morphology (Supplementary Fig. 3C), reminiscent of the original tumours (Fig. 1G), and this was also confirmed histologically (Supplementary Fig. 3D). The transplanted TGOs stained positive for both neutral and acidic mucins, PAS and Alcian Blue (Fig. 1L, Supplementary 3D). Transplantation of $N f k b 1^{-/-}$TGOs was successful in $90 \%(8 / 9)$ of recipient NSG mice and was even

\footnotetext{
${ }^{1}$ The Walter and Eliza Hall Institute of Medical Research, Parkville, VIC, Australia. ${ }^{2}$ Department of Medical Biology, The University of Melbourne, Parkville, VIC, Australia. ${ }^{3}$ School of Clinical Sciences, Monash University, Clayton, VIC, Australia. ${ }^{4}$ Princess Máxima Center for Pediatric Oncology, Utrecht, CT, The Netherlands. ${ }^{5}$ Department of Surgery, The University of Melbourne, Parkville, VIC, Australia. ${ }^{6}$ Present address: Translational Research Institute Australia, Woolloongabba, QLD, Australia. ${ }^{7}$ Present address: Monash Biomedicine Discovery Institute and Department of Biochemistry and Molecular Biology, Monash University, Clayton, VIC, Australia. ${ }^{8}$ Present address: Cancer Genomics Netherlands, Oncode Institute, Utrecht, CG, The Netherlands. ${ }^{9}$ These authors contributed equally: Tracy L. Putoczki, Lorraine A. O'Reilly. ${ }^{凶}$ email: oreilly@wehi.edu.au Edited by Professor Gerry Melino
} 


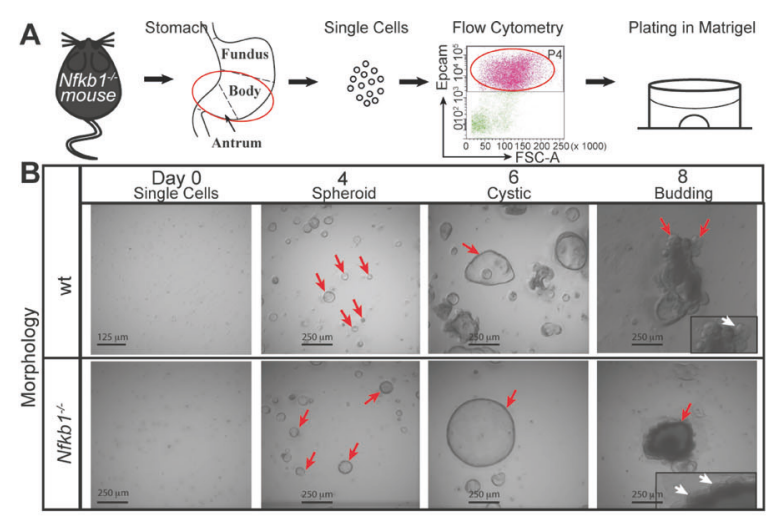

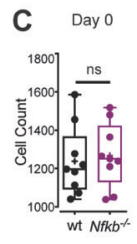

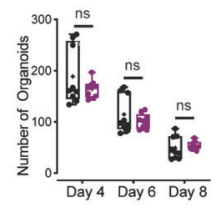

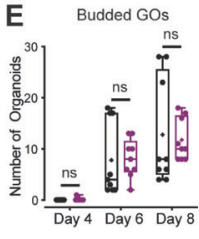

F Budded GOs (\%)

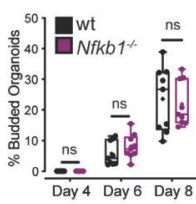

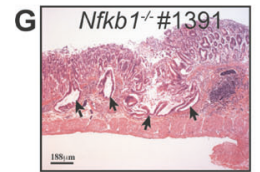

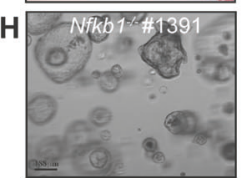

1 \&
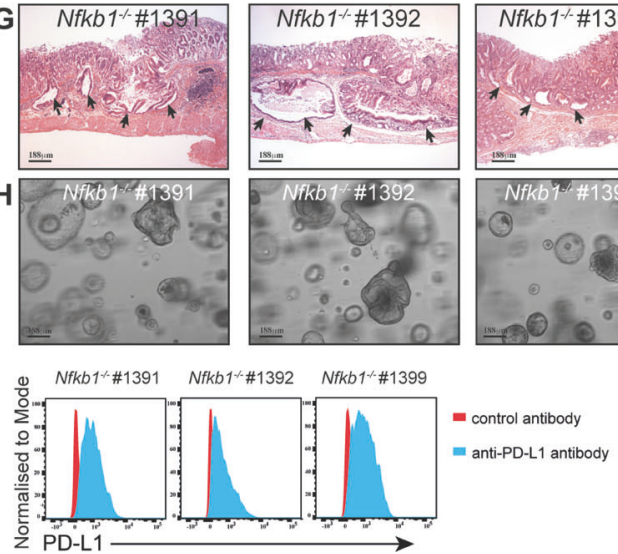
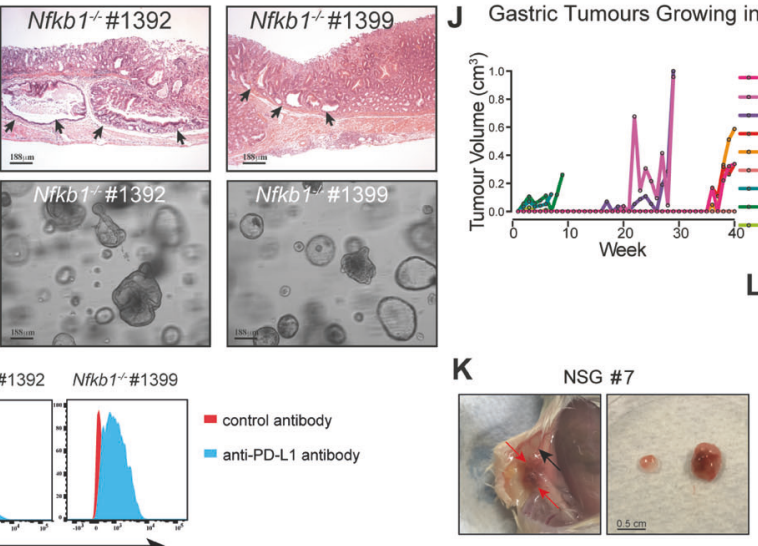

Gastric Tumours Growing in NSG Mice
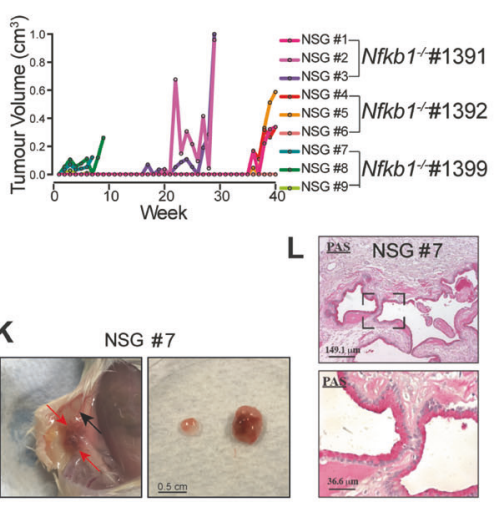

Fig. 1 Establishment of gastroids of pre-neoplastic $\mathrm{Nfkb}^{-/-}$mice and PD-L1 expressing TGOs from GC of old sick $\mathrm{Nfkb}^{-/-}$mice. A Schematic for the culture of gastric epithelial cells. B Representative images of GO cultures showing morphologies (arrows). Graphical representation of $\mathbf{C}$ cell counts of sorted gastric epithelial cells, D GOs observed, inclusive of all spheroid, budding and cystic organoids E budding GOs and $\mathbf{F}$ budding GOs represented as a percentage of total observed GOs. G H\&E stained sections of GCs from Nfkb1 ${ }^{-1-}$ mice used to derive TGOs. Arrows indicate dysplasia or invasion. H Representative images of TGOs established from GC of $N f k b 1^{-1-}$ mice. I Histogram showing PD-L1 expression on cells isolated from $\mathrm{Nfkb}^{-1-}$ TGOs. J Tumor growth from subcutaneous injection of TGOs. Each line represents measurements from a single mouse. $\mathbf{K}$ Representative harvest of subcutaneous tumours derived from injected TGOs. Red arrows $=$ tumour, black arrows = vascularisation. L Representative images of PAS staining (magenta) in transplanted TGOs. C-F Mean $+/=$ SE (student's $T$-test), $N=3$ experimental repeats each with 3 replicates.

observed, albeit with lower frequency (1/6), in young syngeneic $N f k b 1^{-1-}$ recipient mice (Fig. $1 \mathrm{~J}$, Supplementary $3 \mathrm{E}$ ). The reduced TGO uptake in syngeneic $N f k b 1^{-1-}$ is likely due to the presence of a functional immune system ( $T$ and NK cells) not present in NSG mice.

In summary, GOs were generated from the stomachs of young healthy wt and young pre-neoplastic $\mathrm{Nfkb}^{-/-}$mice and TGOs from the GC of sick $\mathrm{Nfkb} 1^{-1-}$ mice. The absence of NF-KB1 results in gastritis in mice with abnormally increased production of proinflammatory cytokines that drive inflammation leading to GC development $[5,6]$. Cytokines can orchestrate a variety of responses in gastrointestinal stem cells, including proliferation and differentiation [10]. The GOs generated could therefore be used for assessing the impact of cytokines on the development of GC $[5,6]$ and to examine whether the loss of NF-kB1 accelerates the acquisition of oncogenic lesions. For instance, we have shown that $\mathrm{Nfkb} 1^{-1-}$ mice that lack TNF have significantly reduced levels of PD-L1 in gastric epithelial cells compared to $N f k b 1^{-1-}$ mice [6]. The GOs and TGOs can be expanded in vitro and frozen, providing a renewable bio-bank for genomic analysis, genetic manipulation, for example by using CRISPR/Cas9 technology [11]. They can also be used for testing novel therapies, particularly those aimed at killing cancer cells, since the TGOs retained expression of markers associated with evasion of immune attack that are relevant to certain human GC subtypes. Injection of TGOs into the stomach serosal layer may provide a more receptive niche for GC growth
[12], including enhanced uptake in $\mathrm{Nfkb}^{-1-}$ or even wt mice for the testing of therapeutic agents.

\section{DATA AVAILABILITY}

The data used to support the findings of this study are available from the corresponding author upon reasonable request.

\section{REFERENCES}

1. Sung H, Ferlay J, Siegel RL, Laversanne M, Soerjomataram I, Jemal A, et al. Global cancer statistics 2020: GLOBOCAN estimates of incidence and mortality worldwide for 36 cancers in 185 countries. CA Cancer J Clin. 2021;71:209-249.

2. Correa P. Human gastric carcinogenesis: a multistep and multifactorial processFirst American Cancer Society Award Lecture on Cancer Epidemiology and Prevention. Cancer Res. 1992;52:6735-40.

3. Pardoll DM. The blockade of immune checkpoints in cancer immunotherapy. Nat Rev Cancer. 2012;12:252-64.

4. Smyth EC, Moehler M. Pembrolizumab in first-line gastric cancer: win, lose, or draw? JAMA Oncol. 2020;6:1539-41.

5. O'Reilly LA, Putoczki TL, Mielke LA, Low JT, Lin A, Preaudet A, et al. Loss of NFkappaB1 causes gastric cancer with aberrant inflammation and expression of immune checkpoint regulators in a STAT-1-dependent manner. Immunity 2018;48:570-83. e8

6. Low JT, Christie M, Ernst M, Drumoutier L, Preaudet A, Ni Y, et al. Loss of NFKB1 results in expression of tumor necrosis factor and activation of signal transducer and activator of transcription 1 to promote gastric tumorigenesis in mice. Gastroenterology 2020;159:1444-58. 
7. Cancer Genome Atlas Research N. Comprehensive molecular characterization of gastric adenocarcinoma. Nature 2014;513:202-9.

8. Lau HCH, Kranenburg O, Xiao H, Yu J. Organoid models of gastrointestinal cancers in basic and translational research. Nat Rev Gastroenterol Hepatol. 2020;17:203-22.

9. Sato T, Vries RG, Snippert HJ, van de Wetering M, Barker N, Stange DE, et al. Single Lgr5 stem cells build crypt-villus structures in vitro without a mesenchymal niche. Nature 2009;459:262-5.

10. Biton $M$, Haber AL, Rogel N, Burgin $G$, Beyaz $S$, Schnell $A$, et al. $T$ helper cell cytokines modulate intestinal stem cell renewal and differentiation. Cell 2018;175:1307-20. e22

11. Drost J, van Boxtel R, Blokzijl F, Mizutani T, Sasaki N, Sasselli V, et al. Use of CRISPR-modified human stem cell organoids to study the origin of mutational signatures in cancer. Science 2017;358:234-8.

12. Busuttil RA, Liu DS, Di Costanzo N, Schröder J, Mitchell C, Boussioutas A. An orthotopic mouse model of gastric cancer invasion and metastasis. Sci Rep. 2018;8:825.

\section{ACKNOWLEDGEMENTS}

We thank Prof Clare Scott for discussions and critical reading of this manuscript, $G$ Siciliano and K Hughes for animal care, B Helbert for genotyping, E Tsui and her team for preparation of histological sections. This work was primarily supported by a Cancer Council New South Wales project grant \#20-13 (LOR, TP) and a Cancer Council Victoria Grant-in-Aid project grant \#1161400 (LOR, AS, TP). Additional support included fellowships and grants from the NHMRC; Canberra; program \#1016701 (AS), program \#487922 (AWB), fellowship; \#1020363 (AS) and project grants; \#1046010 (AS), \#1008614, \#1080498 (TP), a WEHI Dyson Bequest Centenary Fellowship (TP), a Viertel Senior Medical Research Fellowship (TP), an Australian Postgraduate Award (JL and GH), a Top-up Scholarship, Cancer Therapeutics CRC (Australia; JL and GH) and an NHMRC infrastructure grant, Independent Research Institutes Infrastructure Support Scheme Grant \#361646 and the Victorian State Government (OIS grant).

\section{AUTHOR CONTRIBUTIONS}

JTL-study concept and design, acquisition of data, analysis and interpretation of data, drafting of the manuscript, statistical analysis, G-YH-acquisition of data, technical support, MS-technical support, material support, CWT-technical support, material support, LW-technical support, KB-technical support, HYKY - technical support, JFD—study design, $\mathrm{YH}$-material support, JS-critical revision of the manuscript for important intellectual content, AWB-critical revision of the manuscript for important intellectual content, AS-critical revision of the manuscript, intellectual contributions, study supervision, TLP-study concept and design, interpretation of data, critical revision of the manuscript for important intellectual content, study supervision, obtained funding, LAOR-study concept and design, interpretation of data, drafting of the manuscript, study supervision, obtained funding.

\section{COMPETING INTERESTS}

The authors declare no competing interests.

\section{ADDITIONAL INFORMATION}

Supplementary information The online version contains supplementary material available at https://doi.org/10.1038/s41419-021-04376-2.

Correspondence and requests for materials should be addressed to Lorraine A. O'Reilly.

Reprints and permission information is available at http://www.nature.com/ reprints

Publisher's note Springer Nature remains neutral with regard to jurisdictional claims in published maps and institutional affiliations.

\begin{abstract}
Access This article is licensed under a Creative Commons Attribution 4.0 International License, which permits use, sharing, adaptation, distribution and reproduction in any medium or format, as long as you give appropriate credit to the original author(s) and the source, provide a link to the Creative Commons license, and indicate if changes were made. The images or other third party material in this article are included in the article's Creative Commons license, unless indicated otherwise in a credit line to the material. If material is not included in the article's Creative Commons license and your intended use is not permitted by statutory regulation or exceeds the permitted use, you will need to obtain permission directly from the copyright holder. To view a copy of this license, visit http://creativecommons. org/licenses/by/4.0/.
\end{abstract}

(c) The Author(s) 2021 